\title{
Robotic Uterine Manipulator
}

National Cancer Institute

\section{Source}

National Cancer Institute. Robotic Uterine Manipulator. NCI Thesaurus. Code C150420.

A robotic device designed to assist a surgeon in moving the uterus into an optimal position during laparoscopic procedures. 